\title{
On the energy of unit vector fields with isolated singularities
}

\author{
by Fabiano G. B. Brito (São Paulo) \\ and Pawę G. WalczaK (Łódź)
}

\begin{abstract}
We consider the energy of a unit vector field defined on a compact Riemannian manifold $M$ except at finitely many points. We obtain an estimate of the energy from below which appears to be sharp when $M$ is a sphere of dimension $>3$. In this case, the minimum of energy is attained if and only if the vector field is totally geodesic with two singularities situated at two antipodal points (at the "south and north pole").
\end{abstract}

1. Introduction. The energy of any smooth map $F: M \rightarrow N$ between Riemannian manifolds is defined as (see, for example, [2])

$$
E(F)=\frac{1}{2} \int_{M}\|d F\|^{2}
$$

where the integration is performed with respect to the standard measure induced by the Riemannian structure of $M$. Any unit vector field $V$ on $M$ can be considered as a map of $M$ into $T^{1} M$, the unit tangent bundle. The tangent bundle $T M$ (as well as $T^{1} M$ ) carries the natural Riemannian structure (called the Sasaki metric) inherited from the Riemannian structure on $M$ with the use of the Levi-Civita connection $\nabla$. With this Riemannian structure on $T^{1} M$, the energy of any unit vector field $V$ can be expressed by the formula

$$
E(V)=\frac{1}{2} \int_{M}\left(n+\|\nabla V\|^{2}\right)=\frac{n}{2} \operatorname{Vol}(M)+\frac{1}{2} \int_{M}\|\nabla V\|^{2}
$$

where $n=\operatorname{dim} M$ (compare [6]). The last integral in (2), up to some con-

2000 Mathematics Subject Classification: Primary 53C20.

Key words and phrases: energy, vector field, mean curvature, Ricci curvature.

During the preparation of the paper, the first author was supported by CNPq (Brazil), while the second author was supported by FAPESP (Brazil), proj. tem. no. 1999/02684-5. 
stants, is called in [4] the total bending $B(V)$ of $V$ :

$$
B(V)=c_{n} \int_{M}\|\nabla V\|^{2},
$$

where $c_{n}=1 /\left((n-1) \operatorname{Vol}\left(S^{n}\right)\right)$. In [1], [5] and [6] the problem of minimizing $E(V)$ (equivalently, $B(V)$ ) was considered. In [1], the first author proved that the only vector field on $S^{3}$ minimizing energy and/or total bending is (up to congruences) the one tangent to the fibres of the standard Hopf fibration $S^{3} \rightarrow S^{2}$. Wood [6] obtained results which show that this is no more true for Hopf fibrations of spheres $S^{2 n+1}, n>1$. In fact, the problem of minimizing energy on these spheres is still open.

In this paper, we consider the energy of unit vector fields on compact Riemannian manifolds minus finite sets of points (maybe empty). For such vector fields, we get an estimate of energy from below which, in the case of spheres $S^{n}, n>3$, appears to be sharp and is attained by (and only by) totally geodesic fields with two antipodal singularities.

More precisely, we have the following.

THEOREM 1. Let $V$ be a unit vector field on $M \backslash A, M$ being a compact Riemannian manifold of dimension $n+1$ and $A$ a finite subset of $M$.

(i) If $n \geq 2$, then

$$
E(V) \geq \frac{1}{2 n-2} \int_{M} \operatorname{Ricci}(V, V)+\frac{n+1}{2} \operatorname{Vol}(M) .
$$

(ii) If $n \geq 3$, then equality holds in (4) if and only if $V$ is totally geodesic, the $n$-dimensional distribution $D$ orthogonal to $V$ is integrable and defines a Riemannian totally umbilical foliation $\mathcal{F}$.

THEOREM 2. The energy $E(V)$ of any unit vector field with isolated singularities on $S^{n+1}, n \geq 3$, satisfies the inequality

$$
E(V) \geq \frac{n^{2}+n-1}{2(n-1)} \operatorname{Vol}\left(S^{n+1}\right)
$$

and equality holds in (5) if and only if the flow of $V$ consists of great halfcircles connecting two antipodal singularity points ("south and north pole").

The proof of the theorems above is given in Section 3. Section 2 contains two lemmas used in the proof while Section 4 gives some final remarks.

The second author is grateful to the first one for an invitation to the University of São Paulo.

2. Useful lemmas. Let $M$ be a Riemannian manifold, $\operatorname{dim} M \geq 2$, equipped with two complementary distributions $D_{1}$ and $D_{2}$. Let $B_{i}(i=1,2)$ be the (non-symmetric) second fundamental form of $D_{i}$, denote by $A_{i}$ the 
symmetrization of $B_{i}$, by $H_{i}=$ Trace $A_{i}$ the mean curvature vector of $D_{i}$, and by $T_{i}$ the integrability tensor of $D_{i}$ (i.e., $A_{i}(X, Y)=\frac{1}{2}\left(B_{i}(X, Y)\right.$ $\left.+B_{i}(Y, X)\right)$ and $T_{i}(X, Y)=\frac{1}{2}\left(B_{i}(X, Y)-B_{i}(Y, X)\right)$ for $X$ and $Y$ tangent to $\left.D_{i}\right)$. In [3], the following formula was derived:

(6) $\operatorname{div}\left(H_{1}+H_{2}\right)=\left|A_{1}\right|^{2}-\left|H_{1}\right|^{2}-\left|T_{1}\right|^{2}+\left|A_{2}\right|^{2}-\left|H_{2}\right|^{2}-\left|T_{2}\right|^{2}+K\left(D_{1}, D_{2}\right)$, where

$$
K\left(D_{1}, D_{2}\right)=\sum_{i, \alpha} K\left(e_{i} \wedge e_{\alpha}\right)
$$

$K$ is the sectional curvature of $M,\left(e_{i}\right)$ is an orthonormal frame of $D_{1}$ while $\left(e_{\alpha}\right)$ is an orthonormal frame of $D_{2}$. If $D_{1}$ is the 1-dimensional distribution generated by a unit vector field $V$, then $H_{1}=\nabla_{V} V, T_{1} \equiv 0,\left|A_{1}\right|=\left|H_{1}\right|$, $K\left(D_{1}, D_{2}\right)=\operatorname{Ricci}(V, V)$ and $\left|A_{2}\right|^{2}-\left|H_{2}\right|^{2}-\left|T_{2}\right|^{2}=-\sigma_{2}$, where $\sigma_{2}$ is the second mean curvature of $D_{2}$. Therefore, (6) implies directly the following.

Lemma 1. If $V$ is a unit vector field on a Riemannian manifold $M$, then

$$
\operatorname{div}\left(\nabla_{V} V+H\right)=\operatorname{Ricci}(V)-2 \sigma_{2},
$$

where $H$ is the mean curvature vector and $\sigma_{2}$ is the second mean curvature of $D$, the orthogonal complement of the 1-dimensional distribution spanned by $V$.

Assume now that $M$ has bounded geometry (i.e., bounded sectional curvature and injectivity radii $r_{x}, x \in M$, separated away from zero). Let $f: M \backslash A \rightarrow[0, \infty)$ be a function defined on $M$ outside a finite set $A$. Let $x_{0} \in A$ and denote by $S_{r}$ (resp. $B_{r}$ ), $r$ being positive and small, the geodesic sphere (resp., ball) on $M$ of radius $r$ and centre $x_{0}$.

Lemma 2. If $\liminf _{r \rightarrow 0^{+}} \int_{S_{r}} f>0$, then $\int_{M} f^{2}=\infty$.

Proof. Since the geometry of $M$ is bounded there exists $c>0$ such that $\operatorname{Vol} S_{r} \leq c r^{n}, n+1=\operatorname{dim} M$, for sufficiently small $r$. The assumption implies that there exists $\varepsilon>0$ such that

$$
\int_{S_{r}} f \geq \varepsilon
$$

for small $r$. Hölder's inequality implies that

$$
\left(\int_{S_{r}} f^{2}\right)^{1 / 2}\left(\operatorname{Vol} S_{r}\right)^{1 / 2} \geq \varepsilon .
$$

Consequently,

$$
\int_{S_{r}} f^{2} \geq \frac{\varepsilon^{2}}{c r^{n}}
$$


if $r$ is small enough. Again, if $r$ is small, then, by Fubini's Theorem,

$$
\int_{B_{r}} f^{2}=\int_{0}^{r}\left(\int_{S_{t}} f^{2}\right) d t \geq \frac{\varepsilon^{2}}{c} \lim _{\delta \rightarrow 0^{+}} \int_{\delta}^{r} t^{-n} d t=\infty .
$$

3. Proofs of the theorems. Let $V$ be a unit vector field on $M \backslash\left\{x_{1}, \ldots, x_{m}\right\}, M$ being a compact Riemannian manifold of dimension $n+1>2$. Choose a local orthonormal frame $e_{1}, \ldots, e_{n+1}$ in such a way that $e_{n+1}=V$. Denote by $h_{i j}, i, j \leq n$, the components of the second fundamental form of $D$, the $n$-dimensional distribution orthogonal to $V$. Then

$$
\|\nabla V\|^{2}=\left\|\nabla_{V} V\right\|^{2}+\sum_{i, j} h_{i j}^{2} .
$$

An elementary calculation shows that

$$
\begin{aligned}
\sum_{i, j} h_{i j}^{2}= & \frac{1}{n-1} \sum_{i<j}\left(h_{i i}-h_{j j}\right)^{2}+\frac{1}{n-1} \sum_{i<j}\left(h_{i j}+h_{j i}\right)^{2} \\
& +\frac{n-2}{n-1} \sum_{i \neq j} h_{i j}^{2}+\frac{2}{n-1} \sum_{i<j}\left(h_{i i} h_{j j}-h_{i j} h_{j i}\right) .
\end{aligned}
$$

Since the last sum in (10) equals $\sigma_{2}$, the second mean curvature of $D$, (9) and (10) imply the inequality

$$
\|\nabla V\|^{2} \geq \frac{2}{n-1} \sigma_{2} .
$$

If $E(V)=\infty$, there is nothing to prove. So, assume that $E(V)<\infty$, take $r_{k}>0$ small enough and denote by $S_{k}$ and $B_{k}$, respectively, the geodesic sphere and ball of radius $r_{k}$ and centre $x_{k}, k=1, \ldots, m$. Then, by Lemma 1 and the Stokes Theorem,

$$
\begin{aligned}
\left|\int_{M \backslash \bigcup_{k} B_{k}}\left(\operatorname{Ricci}(V, V)-2 \sigma_{2}\right)\right| & =\left|\int_{M \backslash \bigcup_{k} B_{k}} \operatorname{div}\left(\nabla_{V} V+H\right)\right| \\
& =\left|\sum_{k} \int_{S_{k}}\left\langle\nabla_{V} V+H, N_{k}\right\rangle\right| \\
& \leq \sum_{k} \int_{S_{k}}\left\|\nabla_{V} V+H\right\| \leq c_{n} \sum_{k} \int_{S_{k}}\|\nabla V\| .
\end{aligned}
$$

Here, $H$ is the mean curvature vector of $D, N_{k}$ is a unit vector field orthogonal to $S_{k}$ and $c_{n}$ is a constant which depends on $n$ only. Now, Lemma 2 applied to $f=\|\nabla V\|$ yields

$$
\liminf _{r \rightarrow 0^{+}} \int_{S_{r}^{k}}\|\nabla V\|=0, \quad k=1, \ldots, m,
$$


where $S_{r}^{k}$ is the sphere of centre $x_{k}$ and radius $r$. This implies that the integral $\int_{M} \sigma_{2}$ converges and that

$$
2 \int_{M} \sigma_{2}=\int_{M} \operatorname{Ricci}(V, V)
$$

Inequality (4) in Theorem 1 now follows directly from (2), (11) and (13).

From (9) and (10) it follows that, if $n>3$, then equality holds in (11) if and only if $\nabla_{V} V=0, h_{i j}=0$ for all $i \neq j$ and $h_{i i}=h_{j j}$ for all $i, j \leq n$. These conditions are satisfied if and only if $V$ is geodesic, the distribution $D$ is integrable and determines a Riemannian totally umbilical foliation $\mathcal{F}$. This proves Theorem 1 .

To prove Theorem 2, observe that in the case $M=S^{n+1}$, inequality (4) reduces to (5). By the second part of Theorem 1, equality holds in (5) if and only if the foliation $\mathcal{F}$ orthogonal to $V$ is Riemannian and totally umbilical. Leaves of $\mathcal{F}$ are round spheres at a constant geodesic distance from each other. Such spheres have to lie on parallel $(n+1)$-dimensional hyperplanes of $\mathbb{R}^{n+2}$. In that case, the trajectories of $V$ are contained in great circles of $S^{n+1}$ passing through two fixed antipodal points, as claimed in Theorem 2.

4. Final remarks. Theorem 2 establishes, in particular, that

$$
\frac{4 k^{2}+2 k+1}{4 k-2} \operatorname{Vol}\left(S^{2 k+1}\right)
$$

is a lower bound for the energy of non-singular vector fields on $S^{2 k+1}$. Hopf vector fields on odd-dimensional round spheres have energy

$$
\frac{4 k+1}{2} \operatorname{Vol}\left(S^{2 k+1}\right) \text {. }
$$

Therefore, if $k>1$, "north-south" vector fields have less energy than Hopf ones.

It would be interesting to find the infimum for the energy of non-singular unit vector fields on $S^{2 k+1}$. It has to lie in the interval

$$
\left(\frac{4 k^{2}+2 k-1}{4 k-2}, \frac{4 k+1}{2}\right) \text {. }
$$

Note that the problem is completely solved on $S^{3}$ (see [1]), in particular the energies of Hopf vector fields and "north-south" ones are the same. Since the topology of 2-dimensional foliations of $S^{3}$ is pretty well known, one could try to find also the infimum for the energy of those unit vector fields on $S^{3}$ which are orthogonal to integrable distributions. 


\section{References}

[1] F. G. B. Brito, Total bending of flows with mean curvature correction, Differential Geom. Appl. 12 (2000), 157-163.

[2] J. Eells and L. Lemaire, A report on harmonic maps, Bull. London Math. Soc. 10 (1978), 1-68.

[3] P. G. Walczak, An integral formula for a Riemannian manifold with two orthogonal complementary distributions, Colloq. Math. 58 (1990), 243-252.

[4] G. Wiegmink, Total bending of vector fields on Riemannian manifolds, Math. Ann. 303 (1995), 325-344.

[5] - , Total bending of vector fields on the sphere $S^{3}$, Differential Geom. Appl. 6 (1996), 219-236.

[6] C. M. Wood, On the energy of a unit vector field, Geom. Dedicata 64 (1997), 319-330.

Departamento de Matemática-IME-USP

Caixa Postal 66281-CEP 05315-970

São Paulo-SP, Brazil

E-mail: fabiano@ime.usp.br
Department of Mathematics Łódź University Banacha 22 90-238 Łódź, Poland E-mail: pawelwal@imul.uni.lodz.pl 